TRANSACTIONS OF THE

AMERICAN MATHEMATICAL SOCIETY

Volume 339, Number 1, September 1993

\title{
PROBING L-S CATEGORY WITH MAPS
}

\author{
BARRY JESSUP
}

\begin{abstract}
For any map $X \stackrel{f}{\longrightarrow} Y$, we introduce two new homotopy invariants, dcat $f$ and rcat $f$. The classical category cat $f$ is a lower bound for both, while dcat $f \leq$ cat $X$ and rcat $f \leq$ cat $Y$. When $Y$ is an Eilenberg-Mac Lane space, $f$ represents a cohomology class and dcat $f$ often gives a good estimate for cat $X$. We prove that if $\Omega \in H^{n}(M ; \mathbb{Z})$ is the fundamental class of a compact, simply connected $n$-manifold, then dcat $\Omega=$ cat $M$. Similarly, when $X$ is sphere, then $f$ is a homotopy class and while cat $f=1$, rcat $f$ can be a good approximation to cat $Y$. We show that if $\alpha \in \pi_{2}\left(\mathbb{C} P^{n}\right)$ is nonzero, then rcat $\alpha=n$. Rational analogues are introduced and we prove that for $u \in H^{*}(X ; \mathbb{Q})$, dcat $u=1 \Leftrightarrow u^{2}=0$ and $u$ is spherical.
\end{abstract}

\section{INTRODUCTION}

Let Lusternik-Schnirelmann category, cat $X$, of space $X$ is a subtle invariant which is usually difficult to compute. In particular, good lower bounds seem to require much more data than is provided by standard homotopy invariants such as the cohomology ring or homotopy groups. For example, Ginsburg and Toomer [Gi, To] defined lower bounds using the Milnor-Moore spectral sequence, while Felix, Halperin, and Lemairè [Fe-Ha, Ha-Le] employed the full force of the Sullivan model for their approximations.

Much older lower bounds are provided by the category, cat $f$, of a map $X \stackrel{f}{\longrightarrow} Y$, which was introduced originally by Fox [Fo]. Taking his point of view, we introduce two new L-S type invariants for a map $X \stackrel{f}{\longrightarrow} Y$, which we call the domain-category, dcat $f$ and the range category, rcat $f$. These satisfy

$$
\text { cat } f \leq \operatorname{dcat} f \leq \text { cat } X \text { and } \text { cat } f \leq \operatorname{rcat} f \leq \text { cat } Y \text {. }
$$

When specialized to maps representing cohomology (when $Y$ is an EilenbergMac Lane space) or homotopy (when $X$ is a sphere), in many cases these invariants provide excellent approximations to cat $X$ or cat $Y$. In particular, we prove

Theorem 2.8. If $\Omega \in H^{n}(M ; \mathbb{Z})$ is the fundamental class of a simply connected $n$-manifold, then dcat $\Omega=$ cat $M$.

This is to be contrasted with the classical category cat $\Omega$, which is 1 in this case $[\mathrm{Be}-\mathrm{Ga}]$.

Received by the editors May 19, 1989 and, in revised form, June 18, 1991.

1991 Mathematics Subject Classification. Primary 55M30, 55P60, 55 P62.

Key words and phrases. Lusternik-Schnirelmann category, minimal models. 
For homotopy classes $\alpha \in \pi_{n}(X)$, we always have cat $\alpha=1$. However, we show in Example 2.6 that if $\alpha \in \pi_{2}\left(\mathbb{C} P^{n}\right)$; where $\mathbb{C} P^{n}$ is the complex projective space, then rcat $\alpha=n$.

Berstein and Ganea proved that for rational cohomology classes $u \in$ $H^{*}(X, \mathbb{Q})$, cat $u=\min \left\{m \mid u^{m+1}=0\right\}$. By introducing rational analogues of dcat and rcat, we show (Theorem 3.5) that dcat $u$ is at least as large as Toomer's invariant for $u$, which is often larger than cat $u$, particularly when $\operatorname{dim} u$ is odd. We also prove

Theorem 3.8. Suppose $u \in H^{n}(X ; \mathbb{Q})$ is nonzero. Then $\operatorname{dcat}_{0} u=1 \Leftrightarrow u$ is spherical and $u^{2}=0$.

In $\S 2$, we define dcat $f$ and rcat $f$ and show that they may be characterized in terms of the classical category of maps which commute, up to homotopy, with $f$. This allows us to give examples where dcat $f>$ cat $f$, rcat $f>$ cat $f$, and dcat $f \neq \operatorname{rcat} f$. Section 3 studies the rational versions. Using work of Felix and Halperin, we give an algebraic description of dcat $f_{\mathbb{Q}}$ and rcat $f_{\mathbb{Q}}$ for rational maps $f_{\mathbb{Q}}: X_{\mathbb{Q}} \rightarrow Y_{\mathbb{Q}}$. We can then prove some interesting results about the domain and range category of rational cohomology classes.

I am grateful to Stephen Halperin for helpful suggestions, and also to Eadie Henry and Jocelyne Michaud for preparing this manuscript. I also acknowledge the assistance of the National Science and Engineering Research Council of Canada.

\section{DoMAIN AND RANGE CATEGORY}

We restrict ourselves to well-pointed, simply connected spaces with the homotopy type of a CW complex of finite type. For this class of spaces, if $X \stackrel{f}{\longrightarrow} Y$, is a continuous map, we can define cat $f$ as follows, after Whitehead, Berstein, Ganea, and Gilbert [G]: Convert the inclusion of the fat wedge $T^{m+1} Y \rightarrow Y^{m+1}$ into a fibration and pull this back over the diagonal $\Delta_{Y}: Y \rightarrow Y^{m+1}$ to obtain a (Ganea) fibration $p_{m}: E_{m} Y \rightarrow Y$. Then cat $f$ is the least $m$ such that we can lift $f$ to a map $\beta: X \rightarrow E_{m} Y$ so that the diagram below homotopy commutes.

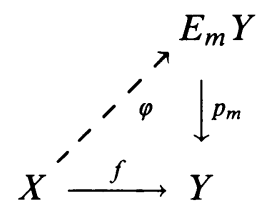

The definitions of domain and range category are variations on this description of cat $f$. Suppose $X \stackrel{f}{\longrightarrow} Y$, and $p_{m}: E_{m} X \rightarrow X$ and $q_{m}: E_{m} Y \rightarrow Y$ are $m$ th-Ganea fibrations for $X$ and $Y$ respectively.

Definition 2.1. The domain-category of $f$, denoted dcat $f$, is the least $m$ such that, in the diagram

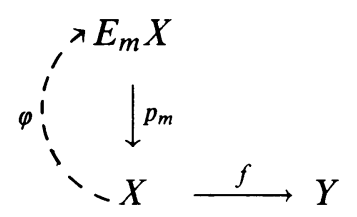

the map $\varphi$ exists so that $f \circ p_{m} \circ \varphi \simeq f$. 
Definition 2.2. The range-category of $f$, denoted rcat $f$, is the least $m$ such that, in the diagram

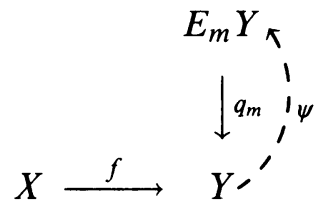

the map $\psi$ exists so that $f \simeq q_{m} \circ \psi \circ f$.

We summarize some straightforward properties of the domain and range categories in the following theorem. All are immediate from the definitions.

Theorem 2.3. For maps $X \stackrel{f}{\longrightarrow} Y$ and $Y \stackrel{g}{\longrightarrow} Z$.

(i) dcat $f$ and rcat $f$ depend only on the homotopy class of $f$.

(ii) cat $f \leq$ dcat $f$ cat $X$ and cat $f \leq$ rcat $f \leq$ cat $Y$.

In particular, cat id $X_{X}=\mathrm{dcat}_{\mathrm{id}}=\mathrm{rcat}_{X}$.

(iii) $\operatorname{dcat}(g \circ f) \leq \operatorname{dcat} f$ and $\operatorname{rcat}(g \circ f) \leq \operatorname{rcat} g$.

A useful characterization of dcat and rcat in terms of the classical category of a map is described in

Theorem 2.4. Suppose $X \stackrel{f}{\longrightarrow} Y$. Then

(i) dcat $f=\min \{$ cat $s \mid X \stackrel{s}{\rightarrow} X$ and $f \circ s \simeq f\}$.

(ii) rcat $f=\min \{$ cat $s \mid Y \stackrel{s}{\rightarrow} Y$ and $s \circ f \simeq f\}$.

Proof. (i) Suppose $X \stackrel{s}{\longrightarrow} X$ with $f \circ s \simeq f$ and cat $s=m$. Now cat $s=m$ implies that there is $\beta: X \rightarrow E_{m} X$ with $p_{m} \circ \beta \simeq s$. Then $f \circ p_{m} \circ \beta \simeq f \circ s \simeq f$ and so dcat $f \leq m$. If, on the other hand, dcat $f=m$, there is $\varphi: X \rightarrow E_{m} X$ with $f \circ p_{m} \circ \varphi \simeq f$. Let $s=p_{m} \circ \varphi$. Then cat $s \leq m$ and $f \circ s \simeq f$. Similar arguments prove (ii).

Example 2.5. Consider the map $h$ defined as the composition $S^{2} \times S^{2} \stackrel{f}{\longrightarrow}$ $S^{4} \stackrel{g}{\longrightarrow} S^{4} \stackrel{g}{\longrightarrow} \mathbb{H} P^{3}$. Here, $f$ extends $S^{2} \vee S^{2} \rightarrow *$ and satisfies $f^{*}\left(\alpha_{4}\right)=$ $\alpha_{2} \times \alpha_{2}^{\prime}\left(\alpha_{2}, \alpha_{2}^{\prime}\right.$ denoting generators in $\left.H^{2}\left(S^{2} \vee S^{2} ; \mathbb{Z}\right)\right)$ and $g$ is the inclusion $S^{4} \rightarrow S^{4} \cup e^{8} \cup e^{12}=\mathbb{H} P^{3}$ into the quaternionic projective space. We show that cat $h=1$, dcat $h=2$, and rcat $h=3$.

Now $H^{*}\left(\mathbb{H} P^{3} ; \mathbb{Z}\right) \cong \mathbb{Z}[u] /\left(u^{4}\right)$ with degree $u=4$. Indeed, if $\tilde{u}: \mathbb{H} P^{3} \rightarrow$ $K(\mathbb{Z}, 4)$ represents $u$ (the latter being an Eilenberg-Mac Lane space), then $S^{4} \stackrel{g}{\longrightarrow} \mathbb{H} P^{3} \stackrel{\tilde{u}}{\longrightarrow} K(\mathbb{Z}, 4)$ also represents $\alpha_{4}$, the generator of $H^{4}\left(S^{4} ; \mathbb{Z}\right)$, since we may choose $u$ so that $g^{*}(u)=\alpha_{4}$. Moreover, estimates from Theorem 1.11 of [Be-Ga] establish cat $\left(S^{2} \times S^{2}\right)=2$, cat $S^{4}=1$, and cat $\mathbb{H} P^{3}=3$. Clearly,

$$
\text { cat } \begin{aligned}
h & =\operatorname{cat}(g \circ f) \leq \min \{\operatorname{cat} f, \text { cat } g\} \\
& \leq \min \left\{\operatorname{cat}\left(S^{2} \times S^{2}\right), \text { cat } S^{4}, \text { cat } \mathbb{H} P^{3}\right\}=1 .
\end{aligned}
$$

If cat $h=0$ then $h$ is homotopic to a constant and so $h^{*} u=0$. But $h^{*} u=$ $f^{*} g^{*} u=f^{*} \alpha_{4}=\alpha_{2} \times \alpha_{2}^{\prime} \neq 0$, and so cat $h=1$.

Let $S^{2} \times S^{2} \stackrel{s_{1}}{\longrightarrow} S^{2} \times S^{2}$ and $\mathbb{H} P^{3} \stackrel{s_{2}}{\longrightarrow} H P^{3}$ be such that $h \circ s_{1} \simeq h, s_{2} \circ h \simeq$ $h$, cat $s_{1}=$ dcat $h$, and cat $s_{2}=\operatorname{rcat} h$. Then cat $s_{1} \leq 2$ and cat $s_{2} \leq 3$. 
Suppose that dcat $h=$ cat $s_{1}=1$. Then

$$
s_{1}^{*}\left(\alpha_{2} \times \alpha_{2}^{\prime}\right)=s_{1}^{*}\left[\left(\alpha_{2} \times 1\right) \cup\left(1 \times \alpha_{2}^{\prime}\right)\right]=s_{1}^{*}\left(\alpha_{2} \times 1\right) \cup s_{1}^{*}\left(1 \times \alpha_{2}^{\prime}\right)=0,
$$

since the cup-length in $s_{1}^{*} \tilde{H}\left(S^{2} \times S^{2} ; \mathbb{Z}\right)$ is at most cat $s_{1}=1$. But $s_{1}^{*}\left(\alpha_{2} \times \alpha_{2}^{\prime}\right)=$ $s_{1}^{*} h^{*} u=h^{*} u=\alpha_{2} \times \alpha_{2}^{\prime}$. Hence cat $s_{1} \geq 2$ and so dcat $h=$ cat $s_{1}=2$.

Similarly, suppose rcat $h=$ cat $s_{2} \leq 2$. Then, as $s_{2}^{*} u=\lambda u$ for some $\lambda \in \mathbb{Z}$, $s_{2}^{*}\left(u^{3}\right)=\lambda^{3} u^{3}=\left(s_{2}^{*} u\right)^{3}=0$ by a cup-length bound. Thus $\lambda=0$ and so $s_{2}^{*} u=0$. But this contradicts $0 \neq \alpha_{2} \times \alpha_{2}^{\prime}=h^{*} u=h^{*} s_{2}^{*} u$ and so we must have rcat $h=3$.

Example 2.6. Consider the complex projective space $\mathbb{C} P^{n}$. Computations as in Example 2.5 show that if $\alpha \in \pi_{2}\left(\mathbb{C} P^{n}\right)$ is the class of a generator, then rcat $\alpha=n$. This is in contrast to the fact that cat $\alpha=1$. Similarly, if $u$ is a generator of $H^{2}\left(\mathbb{C} P^{n}, \mathbb{Z}\right)$, straightforward calculations show that $\operatorname{dcat}\left(u^{k}\right)=n$ for any $k, 1 \leq k \leq n$. However, $\operatorname{cat}\left(u^{k}\right)<n$ for any $k>1$, and in particular, $\operatorname{cat}\left(u^{n}\right)=1$.

Remarks 2.7. These examples show that standard bounds of the form cat $f \leq$ $(\operatorname{dim} X) / p$ for $X \stackrel{f}{\longrightarrow} Y$ (where $Y$ is $p-1$ connected, $p \geq 2$ ) are not valid for dcat or rcat. In Example 2.6, $\mathbb{H} P^{3}$ is 3 connected but dcat $h$ and rcat $h$ both exceed $\left(\operatorname{dim} S^{2} \times S^{2}\right) / p=4 / 4=1$.

The following results show that when applied to cohomology and homotopy classes of a space, dcat and rcat can provide excellent lower bounds for its L-S category.

Theorem 2.8. Suppose $M$ is a compact, simply connected $n$-dimensional manifold and let $\Omega \in H^{n}(M, \mathbb{Z})$ be the fundamental class. Then dcat $\Omega=\operatorname{cat} M$.

Proof. Let $M \stackrel{s}{\longrightarrow} M$ be such that cat $s=\operatorname{dcat} \Omega$ and $s^{*} \Omega=\Omega$. Since $M$ satisfies Poincaré duality, let $D: H^{p}(M, \mathbb{Z}) \rightarrow H_{n-p}(M, \mathbb{Z})$ denote the isomorphism induced by capping with the fundamental homology class $\theta=D(1)$. Since $H_{n}(M, \mathbb{Z})=\mathbb{Z}\langle\theta\rangle$ and $s^{*} \Omega=\Omega$, if $s_{*}$ denotes the induced map on homology, we have $s_{*} D s^{*}=D$. As $D$ is an isomorphism, $s_{*}$ is surjective. But $H_{*}(M, \mathbb{Z})$ is a finitely generated abelian group so $s_{*}$ is an isomorphism. By the Whitehead theorem, $s$ is a homotopy equivalence. But if $s^{\prime}$ is a homotopy inverse, cat $M=$ cat $\mathrm{id}=\operatorname{cat}\left(s^{\prime} \circ s\right) \leq \operatorname{cat} s$ so that cat $s=$ cat $M$. This proves dcat $\Omega=$ cat $M$.

When the structure of a space $X$ depends on more than a single class, we still have

Theorem 2.9. Let $k$ denote $\mathbb{Q}$ or $\mathbb{Z} / p \mathbb{Z}$ with $p$ prime. Let $\left\{u_{i}\right\}$ be a collection of homogeneous generators of $H^{*}(X, k)$ as a $k$-algebra. If $\tilde{u}_{i}: X \rightarrow K\left(k,\left|u_{i}\right|\right)$ represents $u_{i}$ and $u: X \rightarrow \prod_{i} K\left(k,\left|u_{i}\right|\right)$ is $u=\left(\tilde{u}_{1}, \tilde{u}_{2}, \ldots\right)$, then dcat $u \geq$ cat $X_{p}$, where $X_{p}$ is the localization of $X$ at $p$, or the rationalization if $k=\mathbb{Q}$.

Proof. Suppose $X \stackrel{s}{\rightarrow} X$ is such that $\operatorname{dcat} u=\operatorname{cat} s$ and $u \circ s \simeq u$. Then $s^{*} \tilde{u}_{i}=\tilde{u}_{i}$ and so $s^{*}$ is an isomorphism. Then the localized map $s_{p}: X_{p} \rightarrow X_{p}$ is a homotopy equivalence, so cat $s_{p}=$ cat $X_{p}$. But $s_{p}=s \circ l_{p}$ where $l_{p}$ is the localization map $X \rightarrow X_{p}$, and so cat $s_{p} \leq$ cat $s$. Hence dcat $u \geq$ cat $X_{p}$.

An analogous result holds for rcat and homotopy. 
Theorem 2.10. Let $\alpha_{i} \in \pi_{n_{i}}(X)$ generate $\pi_{*}(X)$ and let $A: \bigvee_{i} S^{n_{i}} \rightarrow X$ be the map which is $\alpha_{i}$ on each summand. Then rcat $A=$ cat $X$.

Proof. Any map $X \stackrel{s}{\rightarrow} X$ which commutes with $A$ induces an isomorphism on homotopy groups and so is a homotopy equivalence. This implies that dcat $A=$ cat $X$.

\section{RATIONAL CATEGORY FOR MAPS}

In [Fe-Ha], Felix and Halperin used Sullivan's minimal models to provide a useful algebraic characterization of cat $X$ when $X$ is a rational space. We will define the rational domain and range categories of a map using their methods.

For the homotopy theory of commutative graded differential algebras (CGDAs), we refer the reader to an excellent summary in [Fe-Ha] and for complete details to [Ha1] or [B-G]. For our purposes, we recall the following.

Sullivan defined a functor $A$ which associates to each space $X$ the CGDA over $\mathbb{Q},(A(X), d)$, which consists of the compatible rational differential forms on the singular simplices of $X$. This CGDA computes the rational cohomology of $X: H(A(X), d) \cong H(X ; \mathbb{Q})$. Any continuous map $X \stackrel{f}{\longrightarrow} Y$ gives a CGDA morphism $(A(X), d) \stackrel{A(f)}{\longleftarrow}(A(Y), d)$. If $(A, d) \stackrel{\varphi}{\longrightarrow}(B, d)$ is a morphism of CGDAs with $H^{0}(A)=H^{0}(B)=\mathbb{Q}$, then a Sullivan minimal model of $\varphi$ is a diagram

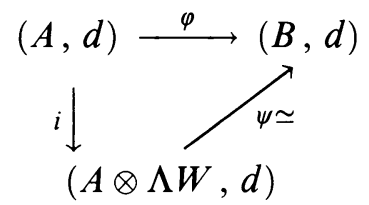

where the $\simeq$ indicates that $\psi$ is an isomorphism on cohomology and $(A, d) \stackrel{i}{\rightarrow}$ $(A \otimes \Lambda W, d)$ is a KS extension as defined in [Ha 1]. In particular, $i(a)=a \otimes 1$ and the graded vector space $W$ has a well ordered, homogeneous basis $\left\{w_{\alpha} \mid \alpha \in\right.$ I\} such that $d w_{\alpha} \in A \otimes \Lambda W_{<\alpha}$ and $\alpha<\beta \Rightarrow \operatorname{deg} w_{\alpha} \leq \operatorname{deg} w_{\beta}$. Here, $\Lambda W$ is the free CGA on $W$ and $W_{<\alpha}$ denotes $\operatorname{span}\left\{w_{\beta} \mid \beta<\alpha\right\}$. The diagram is determined up to isomorphism by $\varphi$ and we say that $i$ represents $\varphi$.

If $X$ is a space, the Sullivan minimal model of $(\mathbb{Q}, 0) \rightarrow(A(X), d)$ (obtained from a basepoint $)$ is of the form $(\mathbb{Q}, 0) \rightarrow(\Lambda W, d) \cong(A(X), d)$, and $(\Lambda W, d)$ is called the Sullivan minimal model of $X$. If $X \stackrel{f}{\longrightarrow} Y$ is a map, then a standard lifting lemma [Ha1, Theorem 5.19] applied to $A(f)$ gives a unique homotopy class of morphisms $\left(\Lambda W_{Y}, d\right) \stackrel{\varphi}{\longrightarrow}\left(\Lambda W_{X}, d\right)$ between minimal models, any of which is called a Sullivan representative of $f$.

Sullivan proved that $\left(\Lambda W_{X}, d\right)$ carries the rational homotopy type of $X$ (i.e., the homotopy type of $X_{\mathbb{Q}}$ ). In particular, besides computing the rational cohomology of $X$, we have, as graded vector spaces, $W_{X}^{k} \cong \operatorname{Hom}_{\mathbb{Z}}\left(\pi_{k}(X), \mathbb{Q}\right)$. Moreover, every morphism between Sullivan minimal models of spaces induces a unique homotopy class of maps between their localizations at $\mathbb{Q}$, the Sullivan representative of any such may being homotopic to the original morphism.

Now let $m$ be a positive integer, $(\Lambda W, d)$ be a Sullivan model of a space $X$ and $E_{m} X \rightarrow X$ denote the $m$ th Ganea fibration. Felix and Halperin showed 
[Fe-Ha, Proposition 2.7 and Theorem 3.1] that one has a commutative diagram

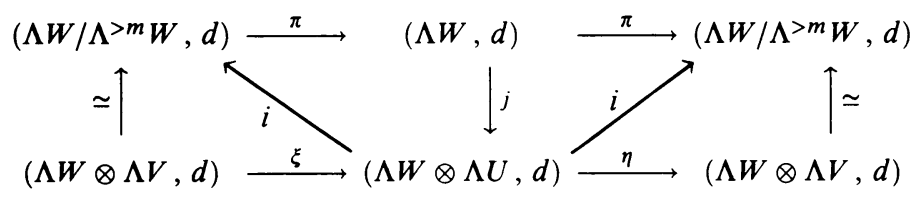

in which $i$ represents the projection $\pi$ and $j$ is a Sullivan representative of $E_{m} X \rightarrow X$. As in [Fe-Ha], define cat $_{0} X$ to be the least integer $m$ such that there is a morphism of CGDAs $\Lambda W \otimes \Lambda V \stackrel{r}{\rightarrow} \Lambda W$ satisfying $r \circ i \simeq \mathrm{id}_{\Lambda} W$. ( $R$ is called a retract.) The above establishes one of their main results, namely, that cat $_{0} X=$ cat $X_{\mathbb{Q}}$.

In this spirit, let $X \stackrel{f}{\rightarrow} Y$ be a map of spaces and suppose

$$
(\Lambda T, d) \stackrel{\varphi}{\longrightarrow}(\Lambda S, d)
$$

is a Sullivan representative of $f$. Consider the diagram

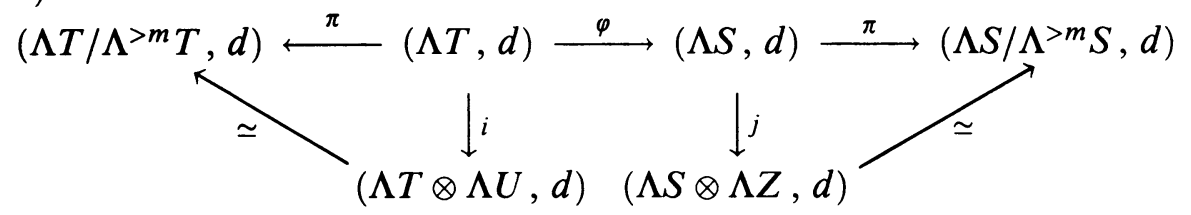

where $i$ and $j$ are Sullivan representatives of Ganea projections.

Definition 3.3. (i) $\mathrm{cat}_{0} f$ is the least $m$ such that a CGDA morphism

$$
(\Lambda T \otimes \Lambda U, d) \stackrel{r}{\longrightarrow}(\Lambda S, d)
$$

exists satisfying $r \circ i \simeq \varphi$.

(ii) dcat $_{0} f$ is the least $m$ such that a CGDA morphism

$$
(\Lambda S \otimes \Lambda Z, d) \stackrel{r}{\longrightarrow}(\Lambda S, d)
$$

exists satisfying $r \circ j \circ \varphi \simeq \varphi$.

(iii) rcat $_{0} f$ is the least $m$ such that a CGDA map

$$
(\Lambda T \otimes \Lambda U, d) \stackrel{r}{\longrightarrow}(\Lambda T, d)
$$

exists satisfying $\varphi \circ r \circ i \simeq \varphi$.

Remark. If $(\Lambda T, d) \stackrel{\varphi}{\longrightarrow}(\Lambda S, d)$ is a CGDA morphism then $\operatorname{cat}_{0} \varphi$, $\operatorname{dcat}_{0} \varphi$, and rcat $_{0} \varphi$ can also be defined by the above.

Now, if $X_{\mathbb{Q}}: f_{\mathbb{Q}} \rightarrow X_{\mathbb{Q}}$, is a localization of $X \stackrel{f}{\rightarrow} Y$, we have, (referring to (3.2) when necessary)

\section{Proposition 3.4.}

(i) cat $_{0} f=$ cat $f_{\mathbb{Q}} \leq$ cat $f$.

(ii) dcat $_{0} f=$ dcat $f_{Q} \leq$ dcat $f$.

(iii) rcat $_{0} f=$ rcat $f_{Q} \leq$ rcat $f$.

(iv) cat $_{0} f \leq \operatorname{dcat}_{0} f$ and cat $_{0} f \leq \operatorname{rcat}_{0} f$.

(v) $\operatorname{dcat}_{0} f=\min \left\{\operatorname{cat}_{0} s \mid(\Lambda T, d) \stackrel{s}{\longrightarrow}(\Lambda T, d)\right.$ satisfies $\left.s \circ \varphi \simeq \varphi\right\}$.

(vi) rcat $_{0} f=\min \left\{\operatorname{cat}_{0} s \mid(\Lambda S, d) \stackrel{s}{\longrightarrow}(\Lambda S, d)\right.$ satisfies $\left.\varphi \circ s \simeq \varphi\right\}$. 
Proof. (i) If we localize the diagram given by cat $f \leq m$ at $\mathbb{Q}$, and note that $\left(E_{m} Y\right)_{\mathbb{Q}} \simeq E_{m} Y_{\mathbb{Q}}$, we obtain one establishing cat $f_{\mathbb{Q}} \leq m$. Then, any Sullivan representative of $X_{\mathbb{Q}} \stackrel{\beta}{\longrightarrow} E_{m} Y_{\mathbb{Q}}$ (from cat $\left.f_{\mathbb{Q}} \leq m\right)$, precomposed with the map $\xi$ of the $Y$-version of diagram (3.1), gives a morphism showing that cat $_{0} f \leq m$. This establishes cat ${ }_{0} f \leq$ cat $f_{\mathbb{Q}} \leq$ cat $f$. Conversely, a morphism guaranteeing that cat $_{0} f \leq m$, precomposed with the map $\eta$ of diagram (3.1) (for $Y$ ), gives a lift of $f_{\mathbb{Q}}$ to $E_{m} Y_{\mathbb{Q}}$ by Sullivan and so we have cat $f_{\mathbb{Q}} \leq m$. This shows that cat ${ }_{0} f=$ cat $f_{\mathbb{Q}}$.

The proofs of (ii) and (iii) are similar, and parts (iv), (v), and (vi) are clear from the definitions.

Remarks. If $Y$ is rational (so that $Y \simeq Y_{\mathbb{Q}}$ ), then for any map $X \stackrel{f}{\rightarrow} Y$, we see that the cat $f=\operatorname{cat}_{0} f$. In particular, if $u \in[X, K(\mathbb{Q}, n)]$ represents a rational cohomology class, we have cat $u=$ cat $_{0} u$. Using this fact, we easily recover the characterization cat $u=\min \left\{n \mid u^{n+1}=0\right\}$ of Berstein and Ganea for rational cohomology classes.

We now investigate some properties of $\operatorname{dcat}_{0} u$ when $u$ is a cohomology class of $H^{*}(X ; \mathbb{Q})$. Suppose $\left(E_{i}^{p}, d_{i}\right)$ is the Milnor-Moore spectral sequence for $X$ over $\mathbb{Q}$. Then, after Toomer [To], define

$$
e_{0} u \equiv \max \left\{p \mid u \text { can be represented in } E_{\infty}^{p}\right\} .
$$

Toomer's invariant for $X, e_{0} X$, is then just the maximum value of $e_{0} u$ for $u$ in $H^{*}(X ; \mathbb{Q})$. If $(\Lambda W, d)$ is a Sullivan minimal model for $X$, we can compute $e_{0} u$ easily. Indeed, by [Fe-Ha, Proposition 9.1], $e_{0} u \equiv \max \{p \mid u$ has a representative in $\Lambda^{\geq p} W$ \} .

Toomer established the inequality $e_{0} X \leq \operatorname{cat}_{0} X$. On the other hand, it is not always true that $e_{0} u \leq$ cat $_{0} u=$ cat $u$. Consider the generator $u_{5} \in$ $H^{5}\left(S^{2} \times S^{3} ; \mathbb{Q}\right)$. Since $u_{5}=\left(u_{2} \times 1\right) \cup\left(1 \times u_{3}\right)$ where $u_{2}$ and $u_{3}$ are generators of $H^{2}\left(S^{2}, \mathbb{Q}\right)$ and $H^{3}\left(S^{3}, \mathbb{Q}\right)$, we see that $e_{0} u_{5} \geq 2$. Moreover, since $\operatorname{cat}\left(S^{2} \times S^{2}\right)=2$, this means that $e_{0} u_{5}=2$. But, as $u_{5}^{2}=0$, we have cat $u=1$. However, the rational domain category of a cohomology class does satisfy such an inequality.

Theorem 3.5. If $u \in H^{n}(X ; \mathbb{Q})$, then $e_{0} u \leq \operatorname{dcat}_{0} u$.

Proof. Suppose $\operatorname{dcat}_{0} u \leq m$. Then we have a diagram

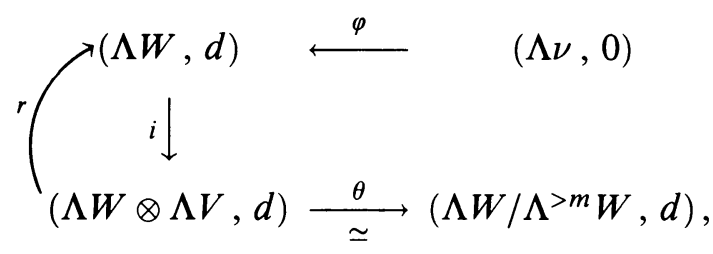

with $r \circ i \circ \varphi \simeq \varphi$, and $\varphi(\nu)$ is some cocycle in $\Lambda W$ with $[\varphi(\nu)]=u$. If $e_{0} u>m$, we may choose $\varphi(\nu) \in \Lambda^{\geq m+1} W$. Now, $u=\varphi^{*}[\nu]=r^{*} i^{*} u$. But $\theta i \varphi(\nu)=0$, so $\theta^{*} i^{*} u=0$; however, $\theta^{*}$ is an isomorphism so we conclude $i^{*} u=0$. This contradicts $u=r^{*} i^{*} u$ unless $u$ is zero, in which case $e_{0} u=0$. In either case, $e_{0} u \leq m$.

Remark. It is possible to have $e_{0} u<\operatorname{dcat}_{0} u$. Let $L=\left(\mathbb{C} P^{2} \vee S^{2}\right) \cup_{\omega} e^{7}$ (where $\omega=[\alpha, \beta]$ for $\alpha \in \pi_{5}\left(\mathbb{C} P^{2}\right), \beta \in \pi_{2}\left(S^{2}\right)$ be Lemaire and Sigrist's 
space [Le]. A straightforward computation, using for example the model for $L$ in [Fe-Ha], shows that if $\Omega \in H^{7}(L)$ is a generator, then $e_{0} \Omega=2$ but $\operatorname{dcat}_{0} \Omega=\operatorname{dcat} \Omega=3$.

A space $X$ is rationally elliptic if both $\operatorname{cat}_{0} X$ and $\operatorname{dim}\left(\pi_{*}(X) \otimes \mathbb{Q}\right)$ are finite. For these spaces, Halperin [Ha2] has shown that $H^{*}(X ; \mathbb{Q})$ is a Poincaré duality algebra. We have the

Theorem 3.6. Suppose $H^{*}(X ; \mathbb{Q})$ satisfies Poincaré duality, and let $\Omega \in$ $H^{n}(X ; \mathbb{Q})$ be the fundamental class. Then $\operatorname{dcat}_{0} \Omega=\operatorname{cat}_{0} X$.

Proof. Any map $X \stackrel{s}{\rightarrow} X$ which satisfies $s^{*} \Omega=\Omega$ is and isomorphism on rational cohomology, and hence $\operatorname{cat}_{0} s=\operatorname{cat}_{0} X$. This shows that $\operatorname{dcat}_{0} \Omega=$ cat $_{0} X$.

Remark. Whether or not we can always attain cat $_{0} X$ with a single cohomology class in the case when $\operatorname{dim}\left(\pi_{*}(X) \otimes \mathbb{Q}\right)=\infty$ is open. However, in all cases checked by the author, it has been possible. (For example, Lemaire's space $L$ is not rationally elliptic but $\operatorname{dcat}_{0} \Omega=$ cat $_{0} L=$ cat $L=3$.)

If $u^{2}=0$ for a nonzero rational cohomology class, then cat c $_{0} u=1$. In the following, we show that this remains true for dcat $_{0}$ if and only if $u$ is a spherical class. By this we mean that there is $S^{n} \stackrel{\gamma}{\rightarrow} X$ so that $S^{n} \stackrel{\gamma}{\rightarrow} X \stackrel{u}{\rightarrow} K(\mathbb{Q}, n)$ represents a generator in $H^{n}\left(S^{n} ; \mathbb{Q}\right)$. Equivalently, $u \in H^{*}(X ; \mathbb{Q})$ is spherical if $e_{0} u=1$. First, we prove

Lemma 3.7. Suppose $(\Lambda V, d) \stackrel{i}{\rightarrow}(\Lambda V \otimes \Lambda W, d)$ is a KS extension such that there is $k \geq 1$ with (a) $e_{0}(\Lambda V, d) \leq k$ and (b) $d: W \rightarrow \Lambda^{\geq k+1}(V \oplus W)$. Then cat $_{0} i=\operatorname{dcat}_{0} i \leq \operatorname{cat}_{0}(\Lambda V, d) \leq \operatorname{cat}_{0}(\Lambda V \otimes \Lambda W)$.

Proof. We construct $(\Lambda V \otimes \Lambda W, d) \stackrel{\varphi}{\longrightarrow}(\Lambda V, d)$ extending the identity on $(\Lambda V, d)$ as follows. Let $\left\{w_{\alpha} \mid \alpha \in I\right\}$ be a KS basis for $W$. Then if 0 is the first element of $I$, we have $d w_{0} \in \Lambda^{\geq k+1} V$. But since $e_{0}(\Lambda V, d) \leq k$, $d w_{0}$ is already a boundary in $\Lambda V$, say $d w_{0}=d b$ for $b \in \Lambda V$. Define $\varphi w_{0}=b$. Then $\varphi$ commutes with the differential. Now assume $\varphi$ has been defined on $\Lambda V \otimes \Lambda W_{<\alpha}$. To define $\varphi\left(w_{\alpha}\right)$ note that $d w_{\alpha} \in \Lambda^{\geq k+1}(V \oplus W)$ and so $\varphi\left(d w_{\alpha}\right) \in \Lambda^{\geq k+1} V$, which allows us to define $\varphi\left(w_{\alpha}\right)$ so as to satisfy $d \varphi\left(w_{\alpha}\right)=\varphi\left(d w_{\alpha}\right)$. Now define $\Lambda V \otimes \Lambda W \stackrel{s}{\rightarrow} \Lambda V \otimes \Lambda W$ by $s=i \circ \varphi$.

Then $s \circ i=i \circ \varphi \circ i=i$ since $\varphi$ extends $\mathrm{id}_{\Lambda V}$. This shows that $\mathrm{dcat}_{0} i \leq$ cat $_{0} s \leq \min \left\{\right.$ cat $_{0} i$, cat $\left._{0} \varphi\right\} \leq$ cat $_{0}(\Lambda V, d)$. Moreover, since $\operatorname{id}_{\Lambda V}=\varphi \circ i$, $\operatorname{cat}_{0}\left(\operatorname{id}_{\Lambda V}\right)=\operatorname{cat}_{0}(\Lambda V, d) \leq \operatorname{cat}_{0} \varphi \leq \operatorname{cat}_{0}(\Lambda V \otimes \Lambda W)$.

Now the promised

Theorem 3.8. Suppose $u \in H^{n}(X ; \mathbb{Q})$ is nonzero. Then $\operatorname{dcat}_{0} u=1 \Leftrightarrow u$ is spherical $u^{2}=0$.

Proof. If $\mathrm{dcat}_{0} u=1$, then cat $_{0} u=1$ and so $u^{2}=0$. Moreover, by Theorem 3.5, $e_{0} u=1$. Thus, $u$ is spherical.

Now suppose $u$ is spherical and $u^{2}=1$. Let $(\Lambda \nu, 0) \stackrel{\varphi}{\longrightarrow}(\Lambda W, d)$ be a Sullivan representative for $u$ with $\operatorname{deg} \nu=n$ and $[\varphi \nu]=u$. As $u$ is spherical, $\varphi \nu=\omega+b$ with $0 \neq \omega \in W$ and $b \in \Lambda^{\geq 2} W$. Consider the case when $n$ is even: since $u^{2}=0$, there is $c \in \Lambda W$ with $d c=(\omega+b)^{2}$; in particular $c=c_{1}+c_{2}$ with $0 \neq c_{1} \in W$ and $c_{2} \in \Lambda^{\geq 2} W$ since $d: W \rightarrow \Lambda^{\geq 2} W$. Define a CGDA $(\Lambda(\nu, x), d)$ by $d \nu=0$ and $d x=\nu^{2}$ (this is the Sullivan model 
of $S^{n}$ if $n$ is even) and extend $\varphi$ to a map $\Phi:(\Lambda(\nu, x), d) \rightarrow(\Lambda W, d)$ by setting $\Phi(x)=c$. Now let

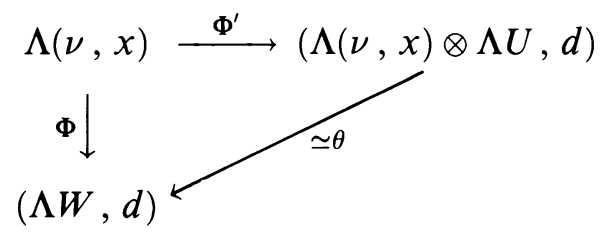

be a Sullivan minimal model for $\Phi$. Since $\Phi$ is injective on homotopy, $d: U \rightarrow$ $\Lambda^{\geq 2}(\langle\nu, x\rangle \oplus U)$. Apply Lemma 3.7 with $k=1$ to $\Phi^{\prime}$. This shows that dcat $_{0} \Phi^{\prime}=1$. But since $(\Lambda(\nu, x) \otimes \Lambda U, d)$ is also minimal, the map $\theta$ above is actually an isomorphism, so $\operatorname{dcat}_{0} \Phi=\mathrm{dcat}_{0} \Phi^{\prime}=1$. But $\varphi$ factors as $\Phi \circ j$ where $(\Lambda(\nu, 0)) \stackrel{j}{\rightarrow}(\Lambda(\nu, x), d)$. Thus $\operatorname{dcat}_{0} \varphi \leq \operatorname{dcat}_{0} \Phi=1$. Thus dcat $_{0} u=1$. The case $n$ odd is handled similarly, but we do not need $x$, as $e_{0}(\Lambda(\nu, 0))=1$ in that case.

We may generalize this to

Theorem 3.9. Suppose $f: X \rightarrow \bigvee_{\alpha} S^{n_{\alpha}}$ induces a surjective map on rational homotopy groups. Then $\mathrm{dcat}_{0} f \leq 1$.

Proof. Let $(\Lambda W, d) \stackrel{\varphi}{\longleftarrow}(\Lambda Z, d)$ be a Sullivan representative of $f$. Now suppose

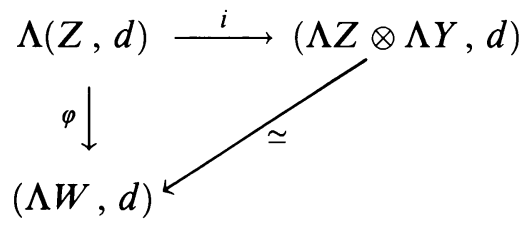

is a Sullivan minimal model of $\varphi$. Since $\pi_{*} f \otimes \mathbb{Q}$ is onto, the derivative in $(\Lambda Z \otimes \Lambda Y, d)$ satisfies $d: Y \rightarrow \Lambda^{\geq 2}(Z \oplus Y)$. Moreover, cat ${ }_{0}\left(\bigvee_{\alpha} S^{n_{\alpha}}\right)=$ $e_{0}\left(\bigvee_{\alpha} S^{n_{\alpha}}\right)=1$, so we may apply Lemma 3.7 with $k=1$ to obtain dcat $_{0} i \leq 1$. But $i$ is also a Sullivan representative of $f$, because $(\Lambda Z \otimes \Lambda Y, d)$ is minimal, thus we may conclude that $\operatorname{dcat}_{0} f \leq 1$.

\section{BIBLIOGRAPHY}

[Be-Ga] I. Berstein and T. Ganea, The category of a map and of a cohomology class, Fund. Math. 50 (1961/62), 265-279.

[B-G] A. K. Bousfield and V. K. A. M. Gugenheim, On PL De Rham theory and rational homotopy type, Mem. Amer. Math. Soc., vol. 8, no. 179, 1976.

[Fe-Ha] Y. Felix and S. Halperin, Rational L.-S. category and its applications, Trans. Amer. Math. Soc. 273 (1982), 1-37.

[Fo] R. H. Fox, On the Lusternik-Schnirelmann category, Ann. of Math. (2) 42 (1941), 333-370.

[G] W. J. Gilbert, Some examples for weak category and conilpotency, Illinois J. Math. 12 (1968), 421-432.

[Gi] M. Ginsburg, On the L.S. category, Ann. of Math. (2) 77 (1963), 538-551.

[Ha1] S. Halperin, Lectures on minimal models, Mém. Soc. Math. France (N.S.) 9-10 (1983).

[Ha2] _ Finiteness in the minimal models of Sullivan, Trans. Amer. Math. Soc. 230 (1977), 173-199.

[Ha-Le] S. Halperin and L.-M. Lemaire, Notions of category in differential algebra, Lecture Notes in Math., vol. 1318, Springer-Verlag, Berlin and New York, pp. 138-154. 
[Le] J. M. Lemairè and F. Sigrist, Sur les invariants d'homotopie rationelle lié à la L.S. catégorie, Comment. Math. Helv. 56 (1981), 103-122.

[To] G. H. Toomer, Lusternik-Schnirelmann category and the Moore spectral sequence, Math. Z. 138 (1974), 175-180.

Department of Mathematics, University of Ottawa, 585 King Edward, Ottawa, OnTARIO, CANADA K1N 6N5

E-mail address: bjessup@acadvm1.uottawa.ca 